\title{
Sur les fonctions harmoniques conjuguées et les séries de Fourier.
}

\section{Par}
A. Kolmogor off (Moscou).

M. Prival off $\left.{ }^{1}\right)$ a démontré le théorème suivant:

Si $f(\theta)$ est une fonction sommable, si de plus

$$
f(\rho, \theta)=\frac{1}{2 \pi} \int_{-\pi}^{+\pi} f(\alpha) \frac{1-\varrho^{2}}{1+\rho^{2}-2 \rho \cos (\alpha-\theta)} d \alpha,
$$

alors, $z$ tendant vers $e^{i \theta}$ le long d'un chemin quelconque non tangent à la circonférence, la fonction harmonique $g(z)$ conjuguée à $f(z)$ tend pour presque toutes les valeurs de $\theta$ vers une limite déterminée

$$
g(\theta)=-\frac{1}{2 \pi} \int_{-\pi}^{+\pi} \frac{f(\theta+\alpha)}{t g \frac{\alpha}{2}} d \alpha,
$$

lintégrale étant comprise comme

$$
\lim _{\varepsilon=0} \int_{-\pi}^{-\varepsilon}+\int_{+\varepsilon}^{+\pi}
$$

En général $g(\theta)$ est une fonction non sommable 2 ). Je démontre dans cette note que la fonction $|g(\theta)|^{1-\varepsilon}$ est sommable pour $\varepsilon>0$ (théorème II). Comme une conséquence immédiate, je démontre un théorème (III) sur la convergence en moyenne de la série de Fourier; on peut déduire de ce théorème que toutes les séries de Fourier-Lebesgue convergent en mesure.

1) "L"intégrale de Cauchy" Saratoff, 1919, en russe.

1) Lusin, "L'intégrale et la série trigonométrique", Moscon, 1915, p. 188. 
Théorème I. Considérons l'ensemble $E$ de tous les points $\theta$ pöur lesquels on a $|g(\theta)|>R, R$ étant un nombre arbitraire. Dans ces conditions on $a$ :

$$
\operatorname{Mes} E . R<C \int_{-\pi}^{+\pi}|f(\theta)| d \theta
$$

où $C$ est une constante absolue.

Démonstration. Supposons qu'il n'existe aucune constante $C$ vérifiant les conditions indiquées. Dans ce cas, pour chaque $n$, il existe une fonction $f_{n}(\theta)$, un ensemble $E_{u}$ et une constante $R_{n}$ tels que l'on a: .

1) $g_{n}(\theta)>R_{n}$, si $\theta$ appartient à l'ensemble $E_{n}$,

2) $\operatorname{Mes} \cdot E_{n} \cdot R_{n}>n$

3) $\int_{-\pi}^{+\pi}\left|f_{n}(\theta)\right| d \theta<1$

On peut, de plus, supposer les fonctions $f_{n}(\theta)$ et $g_{n}(\theta)$ continues; on peut, par exemple, poser $\overline{f_{n}}(z)=f_{n}(z \varrho), \overline{g_{n}}(z)=g_{n}(z \varrho)$, ou $\varrho<1$, mais $1-\varrho$ est assez petit pour vérifier les conditions 1$), 2), 3$ ).

On peut démontrer sans peine, en prenant, s'il est nécessaire, plusieurs $n_{k}$ consécutifs égaux entre eux, l'existence d'une suite $n_{1}, n_{2}$ $n_{i}, \ldots, n_{k}, \ldots$, vérifiant les conditions suivantes:

$$
\lim _{k=\infty} n_{k}=\infty,
$$

(A) la série $\sum_{k=1}^{\infty} \operatorname{Mes} E_{n_{k}}$ diverge,
la série $\sum_{k=1}^{\infty} \frac{\operatorname{Mes} E_{n_{k}}}{\sqrt{n_{k}}}$ converge.

Jonc, en posant $a_{k}=\frac{\sqrt{n_{k}}}{R_{n_{k}}}<\frac{\text { Mes } E_{n_{k}}}{\sqrt{n_{k}}}$, on a:

(B) la série $\sum_{k=1}^{\infty} a_{k}$ converge,

(C) $\lim _{k=\infty} a_{k} R_{n_{k}}=\infty$.

Considérons la somme ( $p_{n}$ - nombres entiers).

$$
\sum_{k=1}^{\infty} a_{k} f_{n_{k}}\left(z^{p_{k}}\right)=\varphi(z)
$$


Cette série converge presque partout sur le cercle $\rho=1$ vers une fonction sommable $\varphi(\theta)$, car on a en vertu de 3 ),

$$
\sum_{n=1}^{\infty} \int_{-\pi}^{+\pi}\left|a_{k} f_{n_{k}}\left(e^{i p_{k} \theta}\right)\right| d \theta<\sum_{k=1}^{\infty} a_{k},
$$

et vers l'intégrale de Poisson de cette tonction dans l'intérieur du cercle. La série.

$$
\sum_{k=1}^{\infty} a_{k} g_{n_{k}}\left(Z^{p_{k}}\right)=\psi(Z)
$$

converge vers la fonction conjuguée $\psi(Z)$ à l'intérieur du cercle.

Oi peut déterminer denx suites

$$
\begin{gathered}
p_{1}<p_{2}<\ldots<p_{k}<\ldots \rightarrow \infty \\
\rho_{1}<p_{2}<\ldots<p_{k}<\ldots \rightarrow 1
\end{gathered}
$$

vérifiant les conditions suivantes:

I. Si $E_{k}$ est l'ensemble des points $e^{i \theta}$ tels que les points $e^{\prime p_{k} \theta}$ ap. partiennent à l'ensemble $E_{n_{k}}$ et si $E$ est l'ensemble limite complet des $E_{k}$, on a

$$
\text { Mes } E=2 \pi \text {. }
$$

II. Pour toute valeur de $\theta$, et $1>\rho \geqslant \rho_{k}$, on a:

$$
\begin{gathered}
\left|\sum_{q=1}^{k-1} a_{q} g_{n_{q}}\left[\left(\rho e^{i \theta}\right)^{p_{q}}\right]-\sum_{q=1}^{k-1} a_{q} g_{n_{q}}\left[\left(e^{i \theta}\right)^{p_{q}}\right]\right|<1, \\
\left|a_{k} g_{n_{k}}\left[\left(\rho e^{i \theta}\right)^{p_{k}}\right]-a_{k} g_{n_{k}}\left[\left(e^{(\theta)}\right)^{p_{k}}\right]\right|<1 .
\end{gathered}
$$

III. Pour tous les $\theta$ et $\rho \leqslant \rho_{k}$. on a:

$$
\left|\sum_{e=n+1}^{\infty} a_{e} g_{n_{e}}\left[\left(\rho e^{\prime \theta}\right)_{p}\right]\right|<1 .
$$

En effet: 1) on peut démontrer, que la condition I est remplie, si les nombres $p_{k}$ croissent assez rapidement 1); 2) étañt donnés $p_{1}$, $p_{2}, \ldots, p_{k}$ on peut choisir $\rho_{k}$ tel que la condition II soit remplio

1) La dómonstration est longue; elle pent être basée aur la divergence de la série $A$ et la remarque suivante: les ensembles $E_{1} E_{2} \ldots E_{n-1}$ b́tant mesurablea, on peut choisir $p_{k}$ si grand que l'ensemble $E_{k}$ soit disposé assez uniformement cur La circonffrence, pour rendre les mesures des intersections $E_{1} E_{2} \ldots E_{k-1}$ avec $E_{k}$ proches de produits des menures. 
en vertu de la continuité des fonctions $\left.g_{n}(\theta) ; 3\right)$ la condition III se trouve remplie par le choix des $p_{k+1}, p_{k+2}, p_{k+3} \ldots$ assez grands, pour que $\rho_{k}^{p k+1}, \rho_{k}^{p_{k}+2}, \varrho_{k}^{p_{k}+3} \ldots$ soient assez proches de zéro pour remplir la condition, ear $g_{n}(0)=0$.

Considérons un point $\theta$ de la circonférence appartenant à l'ensemble $E_{k}$.

$$
\begin{aligned}
& \psi\left(\varrho_{k}, \theta\right)=\sum_{q=1}^{k-1} a_{q} g_{n_{q}}\left[\left(\rho_{k} e^{t \theta}\right)^{p_{q}}\right]+a_{k} g_{n_{k}}\left[\left(\varrho_{k} e^{t \theta}\right)^{\boldsymbol{p}_{k}}\right]+ \\
& +\sum_{q=k+1}^{\infty} a_{q} g_{n_{q}}\left[\left(\rho_{k} e^{i \theta)^{p} q}\right]=\sum_{q=1}^{\dot{k}-1} a_{q} g_{n_{q}}\left[e^{i p_{k} \theta}\right]+a_{k} g_{n_{k}}\left[e^{i p_{k} \theta}\right]+\tau,\right. \\
& |\tau|<3, \\
& \psi\left(\varrho_{k-1}, \theta\right)=\sum_{q=1}^{k-1} a_{q} g_{n_{q}}\left[\left(\varrho_{k-1} e^{i \theta}\right)^{p_{k}}\right]+\sum_{q=k}^{\infty} a_{q} g_{n_{q}}\left[\left(\varrho_{k-1} e^{i \theta}\right)^{p_{k}}\right]= \\
& =\sum_{q=1}^{n-1} a_{q} g_{n_{q}}\left[e^{l_{p} \theta}\right]+\tau^{\prime},\left|\tau^{\prime}\right|<2,
\end{aligned}
$$

en vertu de III et II, On a par conséquent:

$$
\left|\psi\left(\rho_{k}, \theta\right)-\psi\left(\rho_{k-1}, \theta\right)\right|<\left|a_{k} g_{n_{k}}\left[e^{t_{k} \theta}\right]\right|-5>\left|a_{k} R_{n_{k}}\right|-5 .
$$

De là il résulte (voir $I$ et (C)), que pour tous les points $\theta$ de l'ensemble $E, \psi(\varrho, \theta)$ ne tend pas vers une limite, $\rho$ tendant vers l'unité. La contradiction avec le résultat de M. Privaloff démontre le théorème.

Théoreme II. Pour $1>\varepsilon>0$, on $a$.

$$
\int_{-\pi}^{+\pi}|g(\theta)|^{1-\varepsilon} d \theta<\frac{C}{\varepsilon} \int_{-\pi}^{+\pi}|f(\theta)| d \theta
$$

C. étant une constante absolue.

La démonstration peut se baser sur la remarquie suivante: la mesure de l'ensemble, sur lequel $|g(\theta)|>R$, est moindre que la mesure de l'ensemble sur lequel $\left|\frac{1}{\theta}\right|>R$, multipliée par $c \int_{-\pi}^{+\pi}|f(\theta)| d \theta$, et la fonction $\left|\frac{1}{x}\right|^{1-\varepsilon}$ est sommable. 
Théoreme III. Si $f(x)$ est une fonction sommable et $S_{n}$ est la somme des $n$ premiers termes de sa série de Fourier, on a

$$
\lim _{n \rightarrow \infty} \int_{0}^{2 n}\left|f(x)-S_{n}(x)\right|^{1-8} d x=10
$$

pour $\left.1>\varepsilon>0^{1}\right)$.

Démonstration. Il est connu, que

$$
S_{n}=\frac{1}{\pi} \int_{-\pi}^{+\pi} \frac{f(x+\alpha)}{\alpha} \sin n \alpha d \alpha+\omega_{n}(x)
$$

où $\omega_{n}(x)$ tend vers zéro uniformement. D'une manière analogue on peut écrire:

$$
S_{n}=\frac{1}{2 \pi} \int_{-\pi}^{+\pi} \frac{f(x+\alpha)}{\operatorname{tg} \frac{\alpha}{2}} \sin n \alpha d \alpha+\omega_{n}^{\prime}(x)
$$

$w_{n}^{\prime} \rightarrow 0$ uniformoment.

Donc:

$$
S_{n}-\omega_{n}^{\prime}=
$$

$$
=\frac{1}{2 \pi} \int_{-\pi}^{+\pi} \frac{f(x+\alpha)}{\operatorname{tg} \frac{\alpha}{2}} \sin n \alpha d \alpha=\frac{\cos n x}{2 \pi} \int_{-\pi}^{+\pi} \frac{f(x+\alpha) \sin n(x+\alpha)}{\operatorname{tg} \frac{\alpha}{2}} d \alpha-
$$

$$
-\frac{\sin n x}{2 \pi} \int_{-\pi}^{+\pi} \frac{f(x+\alpha) \cos n(x+\alpha)}{\operatorname{tg} \frac{\alpha}{2}} d \alpha
$$

les intégrales étant prises comme $\lim _{s=0} \int_{-\pi}^{-t}+\int_{t=}^{+\pi}$

Si $\varphi(x), \psi(x)$ sont les fonctions conjuguées de

$$
f(x) \sin n x, f(x) \cos n x
$$

nous avons:

$$
\begin{gathered}
\int_{-\pi}^{+\pi}\left|S_{n}(x)\right|^{1-\varepsilon} d x<\int_{-\pi}^{+\pi}\left(\left|\frac{\cos n x}{2 \pi} \varphi(x)\right|+\left|\frac{\sin n x}{2 \pi} \psi(x)\right|+\left|\omega_{n}^{\prime}(x)\right|\right) 1-8 d x< \\
<\int_{-\pi}^{+\pi}\left(|\varphi(x)|^{1-\varepsilon}+|\psi(x)|^{1-\varepsilon}+\left|\omega_{n}^{\prime}(x)\right|^{1-8}\right) d x<
\end{gathered}
$$

1) Pour $\varepsilon=0$ cela est inexact; roir - Bteinhaus, "Sur la convergence on moyenne", Comptyrend de l'Acad. de Cracovie, 1918. 
(2)

$$
\begin{gathered}
<\int_{-\pi}^{+\pi}\left(\frac{C}{\varepsilon}|f(x) \sin n x|+\frac{C}{\varepsilon}|f(x) \cos n x|+\left|\omega_{n}^{\prime}(x)^{1-\varepsilon}\right| d x<\right. \\
<\frac{2 C}{\varepsilon} \int_{-\pi}^{+\pi}|f(x)| d x+\int_{-\pi}^{+\pi}\left|\omega_{n}^{\prime}(x)\right|^{1-\varepsilon} d x .
\end{gathered}
$$

On peut pour $h>0$ arbitraire former deux fonctions $f^{\prime}(x), f^{\prime \prime}(x)$, telles qu'on ait:

1. $f^{\prime}(x)+f^{\prime \prime}(x)=f(x)$.

2. $\int_{-\pi}^{+\pi}\left|f^{\prime \prime}(x)\right| d x<\frac{h \varepsilon}{4 C}, \int_{-\pi}^{+\pi}\left|f^{\prime \prime}(x)\right|^{1-\varepsilon} d x<\frac{h}{2}$.

3. $f^{\prime}(x)$ est continue; alors elle vérifie la condition (1). En appliquant la formule (2) à $f^{\prime \prime}(x)$ et en désignant par $S_{n}^{\prime}, S_{n}^{\prime \prime}$ les sommes partielles des séries de Fourier de $f^{\prime}(x), f^{\prime \prime}(x)$, nous avons:

$$
\begin{aligned}
\limsup & \int_{n=\infty}^{+\pi}\left|f(x)-S_{n}(x)\right|^{1-\varepsilon} d x<\lim \sup \int_{-\pi}^{+\pi}\left|f^{\prime}(x)-S_{n}^{\prime}(x)\right|^{1-\varepsilon} d x+ \\
& +\int_{-\pi}^{+\pi}\left|f^{\prime \prime}(x)\right|^{1-\varepsilon} d x+\lim \sup \int_{-\pi}^{+\pi}\left|S_{n}^{\prime \prime}\right|^{1-\varepsilon} d x<h .
\end{aligned}
$$

Cela démontre le théorème:

Moscou, Février 1923. 